\title{
Environmental novelty is associated with a selective increase in Fos expression in the output elements of the hippocampal formation and the perirhinal cortex
}

\author{
Michael VanElzakker, Rebecca D. Fevurly, Tressa Breindel, and Robert L. Spencer ${ }^{1}$ \\ Department of Psychology, University of Colorado at Boulder, Boulder, Colorado 80309, USA
}

\begin{abstract}
If the hippocampus plays a role in the detection of novel environmental features, then novelty should be associated with altered hippocampal neural activity and perhaps also measures of neuroplasticity. We examined Fos protein expression within subregions of rat hippocampal formation as an indicator of recent increases in neuronal excitation and cellular processes that support neuroplasticity. Environmental novelty, but not environmental complexity, led to a selective increase of Fos induction in the final "output" subregion of the dorsal hippocampal trisynaptic circuit (CAl) and a primary projection site (layer five of the lateral entorhinal cortex, ERC), as well as in the perirhinal cortex. There was no selective effect of novelty on Fos expression within "input" elements of the trisynaptic circuit (ERC layer two, the dentate gyrus or CA3) or other comparison brain regions that may be responsive to overall motor-sensory activity or anxiety levels (primary somatosensory and motor cortex or hypothalamic paraventricular nucleus). Test session ambulatory behavior increased with both novelty and environmental complexity and was not significantly correlated with Fos expression patterns in any of the brain regions examined. In contrast, the extent of manipulated environmental novelty was strongly correlated with Fos expression in CAl. These results support the prospect that a novelty-associated signal is generated within hippocampal neurocircuitry, is relayed to cortical projection sites, and specifically up-regulates neuroplasticitysupporting processes with dorsal hippocampal CAl and ERC layer five. Whether novelty-dependent Fos induction in perirhinal cortex depends on this hippocampal output or reflects an independent process remains to be determined.
\end{abstract}

The hippocampus appears to play an essential role in the encoding of configural and temporal relationships between experiential elements thereby supporting memory for environmental contexts and discrete episodes (Rudy and Sutherland 1995). A related hypothesis is that the hippocampus serves as a functional comparator of present and past (stored) experience, and consequently directs attention and mnemonic processes to the novel aspects of present experience (Margulies 1985; Otto and Eichenbaum 1992; Knight 1996; Mizumori et al. 1999; Moser and Paulsen 2001; Vinogradova 2001; Fyhn et al. 2002; Norman and O'Reilly 2003). A comparator capability of the hippocampus seems plausible given the converging parallel neural pathways by which multimodal sensory information is presented to the hippocampus. The entorhinal cortex serves as an anatomical gateway through which the majority of cortically processed information is presented to the hippocampus. This cortical information is relayed directly (via monosynaptic connections) to CA1 neurons (originating primarily from layer three of the entorhinal cortex) or to CA3 neurons (originating primarily from layer two of the entorhinal cortex) (Steward and Scoville 1976; Remondes and Schuman 2004; Witter and Amaral 2004). In addition, CA1 neurons are presented with cortical information (originating primarily from layer two of the entorhinal cortex) that has first been processed by the dentate gyrus and CA3, via the serial connections of the hippocampal formation trisynaptic circuit (Andersen

'Corresponding author.

E-mail robert.spencer@colorado.edu; fax (303) 492-2967.

Article is online at http://www.learnmem.org/cgi/doi/10.1101/lm.1196508. et al. 1971). Although both CA1 and CA3 neurons receive direct and indirect neural input from entorhinal cortex, several hippocampal-circuit models propose that CA1 neurons have unique access to both past (stored) and ongoing experiential neural patterns (Hasselmo and Schnell 1994; Moser and Paulsen 2001; Norman and O'Reilly 2003). Alternatively, other models posit an important role of CA3 neurons (Mizumori et al. 1999; Vinogradova 2001; Lee et al. 2005a) and/or dentate gyrus granule cells (Meeter et al. 2004; Lee et al. 2005a) in the detection of novel features of experience.

Implicit in these models of hippocampal function is the assumption that the hippocampus is engaged differently when presented with novel versus familiar stimuli patterns. There is some evidence for experience-dependent differences in rodent hippocampal activity that are manifest by electrophysiological differences in individual or ensemble neuronal activity patterns (Otto and Eichenbaum 1992; Fyhn et al. 2002; Nitz and McNaughton 2004). Neuroimaging studies in humans have detected increased fMRI activity in the hippocampal region during encoding of novel visual stimuli (Stern et al. 1996; Johnson et al. 2008). Moreover, humans with hippocampal damage exhibit altered event-related potentials in response to novel stimuli (Knight 1996).

Hippocampal activity that varies with the novelty of an experience may be important for guiding ongoing behavior (e.g., exploratory behavior and vigilance), and if so, should also produce detectable differences in activity of hippocampal efferents. In addition, detection of novelty may be important for altering neuroplastic processes within components of the hippocampus. 
The goal of our study was to examine across hippocampal formation subregions the levels of a cellular marker of neural activity and neuroplasticity (Fos expression) associated with environmental experiences that vary in novelty and complexity. The expression of the protein product, Fos, of the immediate early gene, $c$-fos, may be a good molecular indicator of recent increases in general molecular changes that contribute to neuroplasticity. Expression of Fos reflects an intracellular state of cells that varies primarily as a result of recent activation by intercellular signals (e.g., neurotransmitters, hormones, paracrine factors, and adhesion molecules) (Herdegen and Leah 1998). Hippocampal Fos expression is associated with recent increases in neuronal firing, although apparently in a complex fashion (Labiner et al. 1993). Increases in hippocampal Fos is also believed to be an important mediator of activity-dependent neuroplasticity (Sheng and Greenberg 1990).

In our study we examined the number of Fos immunopositive cells in the dentate gyrus, subregions of the hippocampus (CA1, CA2, CA3, and CA4), and layers two and five of the lateral entorhinal cortex. In addition, we examined Fos immunoreactivity in the perirhinal cortex. There is accumulating support for this brain region to play a role in the detection of novel stimuli in a configuration independent manner (Brown and Aggleton 2001; Kumaran and Maguire 2007). For comparison purposes, we also examined Fos expression patterns in primary somatosensory cortex, primary motor cortex, and the hypothalamic paraventricular nucleus (PVN). Fos expression levels in the somatosensory and motor cortex may reflect the varying amounts of somatosensation and motor activity present during the experimental test-day experiences. Fos expression levels in the PVN may reflect the varying amounts of test-day stress and anxiety associated with the different treatment conditions.

Several other rat studies have examined the relationship between stimuli novelty (e.g., visual images, extramaze environmental cues, or new learning tasks) and Fos expression in the hippocampus (Hess et al. 1995a; Wan et al. 1999; Vann et al. 2000). Whereas those other studies utilized tasks that had a training phase and operant reward component, our study examined Fos expression in rats placed in a novel or familiar environment with no training components or operant contingencies. The pattern of Fos expression associated with unrewarded exploratory behavior may better reflect the extent to which novelty and complexity differentially and automatically engage the hippocampus than does the pattern of Fos expression associated with various learning regimens and their particular task demands (Kumaran and Maguire 2007).

\section{Results}

\section{Experiment 1: Variation in environmental complexity}

The first experiment examined whether exposure of rats to a novel environment (arena) with varying complexity (zero, one, or five objects within the arena) produced varying amounts of Fos immunoreactivity within the hippocampus and dentate gyrus. Placement of rats for the first time into the arena produced a large increase in the number of Fos immunoreactive cells in the principal cell layer of the CA1, CA3, and CA4 subdivisions of the dorsal hippocampus and in the suprapyramidal granule cell layer of the dentate gyrus $\left(F_{(3,12)}=9.5-34.6, P<0.01\right.$; Figs. 1 and 2$)$. The amount of Fos immunoreactivity induction was similar regardless of whether the arena was empty or had one or five objects placed on its floor. There was also a trend for the novel exposure to produce an increase in Fos immunoreactivity within the CA2 subdivision of the dorsal hippocampus $\left(F_{(3,12)}=2.7\right.$, $P=0.09$ ).
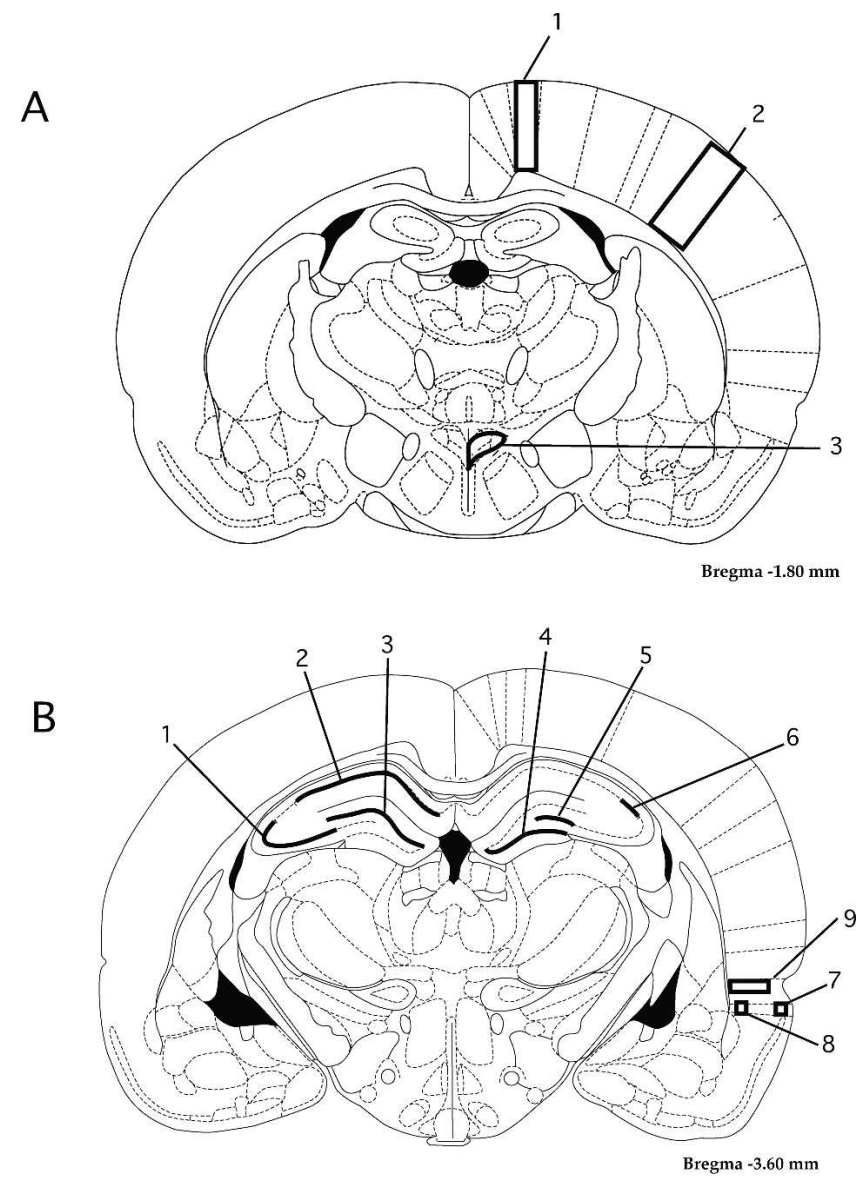

Figure 1. Diagram of approximate regions of interest (ROI) that were selected for determination of the number of Fos immunoreactive cells present in brains after exposure to various treatment conditions. $(A)$ Coronal section ( $1.8 \mathrm{~mm}$ posterior to bregma); labeled ROI are (1) primary motor cortex, (2) primary somatosensory cortex (barrel fields), and (3) hypothalamic paraventricular nucleus. (B) Coronal section $(-3.6 \mathrm{~mm}$ posterior to bregma); labeled ROI are (1) CA3, (2) CA1, (3) dentate gyrus, suprapyramidal blade, (4) dentate gyrus, infrapyramidal blade, (5) CA4, (6) CA2, (7) lateral entorhinal cortex, layer two, (8) lateral entorhinal cortex, layer five, and (9) perirhinal cortex.

\section{Experiment 2: Variation in environmental novelty}

The second experiment examined whether exposure of rats to an environment with varying amounts of novelty (first time in arena, fifth time in arena, or fifth time in arena with one or five novel objects on the floor) produced varying amounts of Fos immunoreactivity within the hippocampus, dentate gyrus, lateral entorhinal cortex, perirhinal cortex, and select other comparison brain regions. In addition, exploratory behavior was monitored on the test day.

\section{Ambulatory behavior}

Overall, rats appeared to actively explore the arena and any objects that it contained. The rats moved throughout the arena and at times, sniffed, pushed, and climbed on the objects. The greatest amount of ambulation occurred during the first 10 min after placement in the arena, and it declined substantially for all groups by the second $10 \mathrm{~min}$ interval (time main effect, $F_{(1,24)}=84.3, P<0.01$ ) (Fig. 3). Rats that were placed in the arena for the fifth time had significantly less overall activity during the first $10 \mathrm{~min}$ than rats placed in the arena for the first time, or rats 


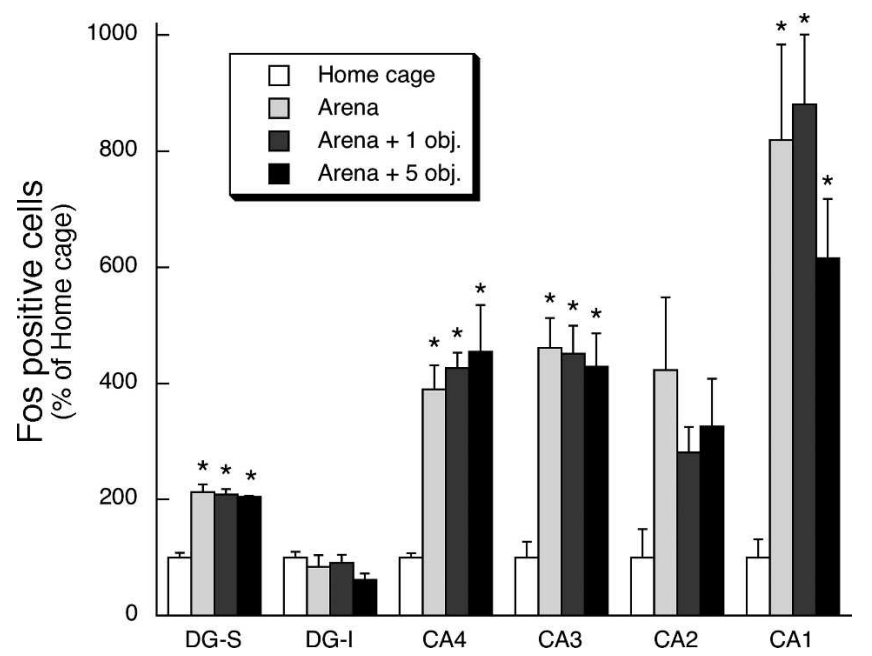

Figure 2. Effect of first time placement in an arena with varying environmental complexity on relative Fos expression within hippocampal subregions. Rats were placed for the first time in a circular arena that contained zero, one, or five objects on its floor. After arena exposure (30 $\mathrm{min}$ ) rats were returned to their home cage for an additional $120 \mathrm{~min}$ Control rats remained in their home cage. ${ }^{*} P<0.01$, compared to homecage control treatment group ( $\mathrm{N}-\mathrm{K}$ test).

placed in the arena for the fifth time with five novel objects placed on the floor [treatment group main effect, $F_{(3,24)}=6.2$, $P<0.01$, and post-hoc comparison, $P<0.05$, Neuman-Keuls test $(\mathrm{N}-\mathrm{K})]$. During the second $10 \mathrm{~min}$, rats that were placed in the arena for the fifth time with five novel objects were significantly more active than the other three treatment groups $(P<0.05$, Fisher's least significant difference).

\section{Fos in hippocampal formation, entorhinal cortex, and perirhinal cortex}

Placement of rats in the arena for the first or fifth time \pm novel objects produced a large increase in the number of Fos immunoreactive cells in the principal cell layer of all hippocampal subdivisions $\left(F_{(4,35)}=4.1-22.5, P<0.01\right)$, lateral entorhinal cortex layers two $\left(F_{(4,35)}=2.7, P<0.05\right)$ and five $\left(F_{(4,35)}=10.5\right.$, $P<0.01)$, and the perirhinal cortex $\left(F_{(4,35)}=16.5, P<0.01\right)$ (Figs. 1 and 4$)$. These test day experiences produced a more modest percent increase in the number of Fos immunoreactive cells in the suprapyramidal granule cell layer of the dentate gyrus $\left(F_{(4,35)}=3.2, P<0.05\right)$. In contrast, these test day experiences produced a decrease in the number of Fos immunoreactive cells in the infrapyramidal granule cell layer of the dentate gyrus $\left(F_{(4,35)}=3.9, P<0.05\right)$.

In contrast to the between-group Fos expression patterns of Experiment 1, in this experiment there were three brain regions in which the magnitude of Fos induction differed between the different arena exposure treatment groups. Rats placed in an empty arena for the first time or placed in an arena for the fifth time with five novel objects had significantly more Fos immunoreactive cells in the CA1 hippocampal subregion than rats placed in the arena for the fifth time without any novel objects $(P<0.05, \mathrm{~N}-\mathrm{K})$ (Figs. 4 and 5). In CA1 there was a striking between-group Fos expression pattern that corresponded to the degree of environmental novelty. The greatest amount of Fos expression was present in the group of rats exposed to the arena for the first time, whereas rats that had prior exposure with the arena exhibited an increasing graded response that depended on whether the arena contained zero, one, or five novel objects on the test day (Figs. 4 and 5). Rats that were placed in the arena for the first time also had significantly more Fos immunoreactive cells in layer five of the lateral entorhinal cortex $(P<0.05$, Fisher's least significant difference) and perirhinal cortex $(P<0.05, \mathrm{~N}-\mathrm{K})$ than rats placed in the arena for the fifth time (Figs. 4 and 6).

Fos in PVN, primary motor cortex, and primary somatosensory cortex Placement of rats in the arena for the first or fifth time \pm novel objects produced a large increase in the number of Fos immunoreactive cells in the hypothalamic paraventricular nucleus $\left(F_{(4,33)}=6.6, P<0.01\right)$, primary somatosensory cortex (barrel fields; $\left.F_{(4,35)}=4.6, P<0.01\right)$, and primary motor cortex $\left(F_{(4,34)}=26.9, P<0.01\right)$. However, the increased Fos expression relative to the home-cage control rats was comparable in all rats placed in the arena regardless of prior exposure to the arena or the number of novel objects present in the arena on the test day (Figs. 1 and 7).

\section{Correlation between Fos and ambulatory behavior or test day novelty condition}

Examination of the group mean Fos expression patterns in the various brain regions (Figs. 4 and 7) suggests that there was not a strong relationship between the amount of Fos expression and the overt activity of rats during their exposure to the arena on the test day (Fig. 3). Rather, for some brain regions described above (CA1, layer five ERC, and perirhinal cortex) there appeared to be a strong relationship between the amount of Fos induction and the extent of environmental novelty that was present during the test session. To further examine these relationships we conducted correlation analyses: (1) between Fos expression levels in a given brain region and total ambulatory behavior during the test session or (2) between Fos expression levels in a given brain region and the ordinal rank of novelty that we expect was asso-

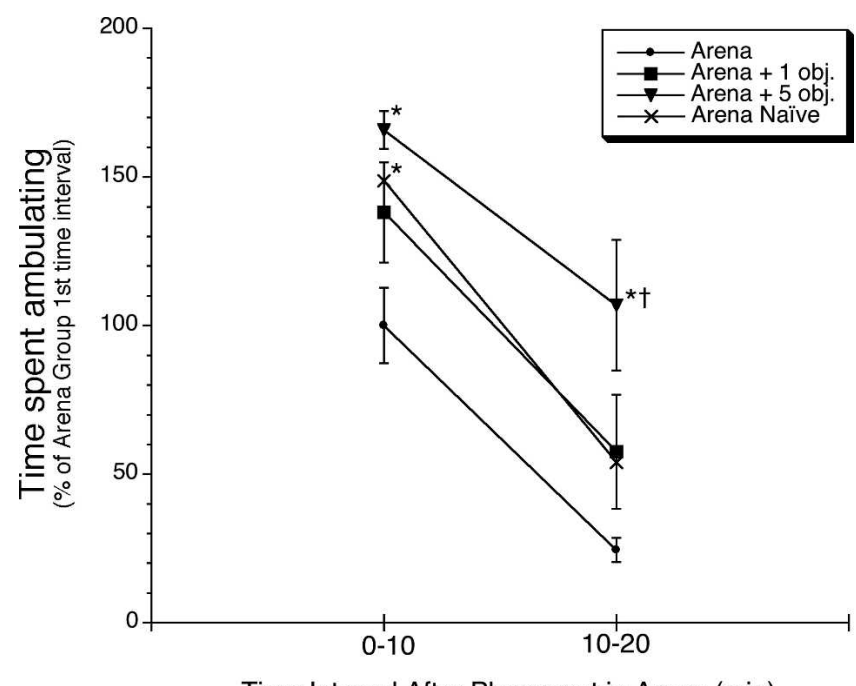

Time Interval After Placement in Arena (min)

Figure 3. Effect of first time placement in arena or fifth time placement in arena (with zero, one, or five novel objects) on ambulatory behavior. Rats were placed for the first time in a circular arena that contained zero objects on its floor ("Naiive" group), or fifth time in the arena with zero, one, or five novel objects on the floor. Ambulatory behavior (see Materials and Methods) was scored for the first and second 10-min intervals while in the arena. ${ }^{*} P<0.05$, compared to rats placed in the arena for the fifth time with zero objects ("Arena" group) during the same time interval (N-K test); $\dagger P<0.05$, compared to the other three treatment groups during the same time interval (Fisher's least significant difference test). 


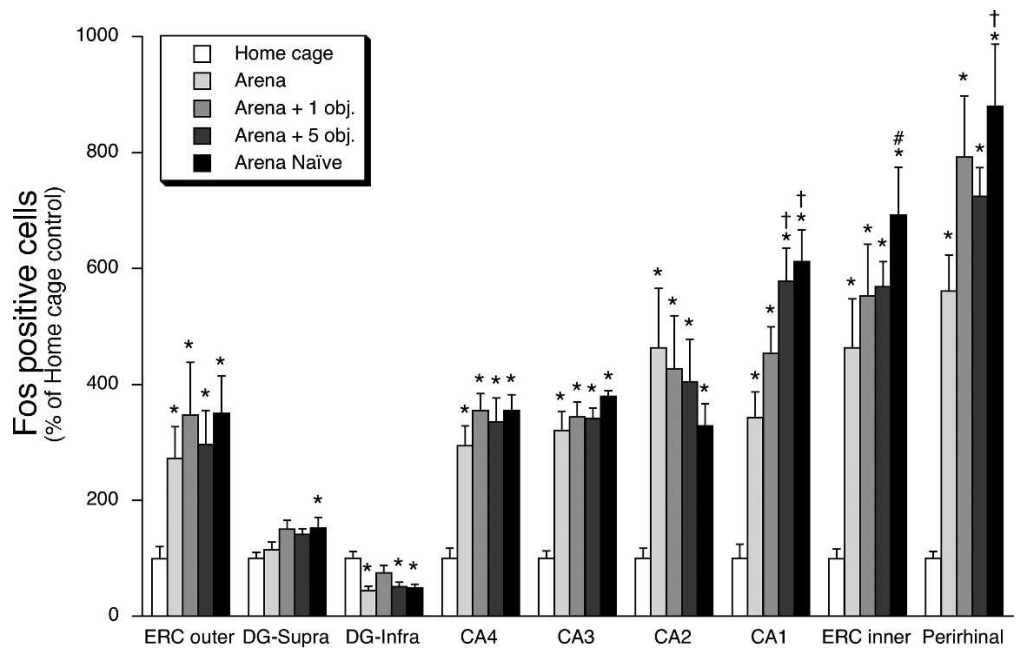

Figure 4. Effect of first time placement in arena or fifth time placement in arena (with zero, one, or five novel objects) on relative Fos expression within hippocampus, entorhinal cortex, and perirhinal cortex. Rats were placed for the first time in a circular arena that contained zero objects on its floor ("Naïve" group), or fifth time in the arena with zero, one, or five novel objects on the floor. After arena exposure $(30 \mathrm{~min})$ rats were returned to their home cage for an additional $120 \mathrm{~min}$. Control rats remained in their home cage. The relative Fos expression in the various subregions is presented in the order left to right that approximates the serial order of neural connections between these subregions, ending with perirhinal cortex that has reciprocal connections with entorhinal cortex (ERC = lateral entorhinal cortex, $\mathrm{DG}=$ dentate gyrus). ${ }^{*} P<0.05$, compared to home-cage control treatment group (N-K test). $\dagger, \# P<0.05$, compared to rats placed in the arena for the fifth time with zero objects ("Arena" group); ( $\dagger, \mathrm{N}-\mathrm{K}$ test; \#, Fisher's least significant difference test).

ciated with each test condition. Our a priori expected extent of novelty present was assigned a rank value of 1-4 (see Materials and Methods, Statistical analysis). There was not a significant correlation between ambulatory activity and Fos expression in any of the brain regions examined (Table 1). In three brain regions examined there was a nominally significant $(P<0.05)$ positive correlation between Fos expression and novelty rank (Table 1). These three brain regions were the same ones that exhibited a significant difference in Fos expression between the different arena exposure conditions-CA1, layer five of ERC, and perirhinal cortex. One of these brain regions, CA1, displayed an especially strong correlation with novelty rank $(r=0.61)$, and this correlation was statistically significant when using the Bonferroni method to control for multiple comparisons $(P<0.002)$.

\section{Discussion}

The key finding of this study was that environmental novelty was associated with a selective increase in Fos expression in the final element of the trisynaptic circuit (CA1 neurons), its projection site (layer five of the entorhinal cortex), and the perirhinal cortex. In contrast, there was no association between Fos expression and environmental novelty in the upstream elements of the trisynaptic circuit (neurons in layer two of the entorhinal cortex, dentate gyrus, and CA3).

Although a wide range of experiences lead to an increase in hippocampal Fos expression, investigators have yet to determine the extent to which Fos expression levels may be associated with certain features of experience. In this study, placement of rats in an arena produced a substantial increase in the number of Fos immunoreactive cells in all brain regions examined, with the one exception being the infrapyramidal blade of the DG (see discussion at the end of this section). The widespread Fos induction in the brains of rats exposed to an experience outside of their home cage compared to very low Fos levels in home-cage control rats is a hallmark characteristic of Fos expression, and has been observed in many other studies (Herdegen and Leah 1998). We found, however, in our first experiment that the number of Fos immunoreactive cells did not vary with the stimulus complexity of the arena setting. Thus, there were equivalent amounts of Fos induction in rats that were placed in an arena containing zero, one, or five objects. This may indicate that hippocampal Fos induction is not related to this particular dimension of stimulus complexity. Another possibility is that the stimulus complexity of the arena itself was sufficient to produce a ceiling level of Fos induction. Vann et al. (2000) observed greater amounts of Fos immunoreactivity in the hippocampus of rats that had been trained and tested to retrieve food pellets on an eight-arm radial arm maze compared to rats that were trained and tested on a single arm of the test apparatus. The differential Fos induction in that study may be associated with differing degrees of spatial complexity; however, an association with different task demands of the two test conditions cannot be ruled out.

In our second experiment we added a novelty dimension, and we extended our Fos expression analysis to additional brain regions. We also examined the ambulatory behavior of each rat during arena exposure on the test day. Exploratory behavior in rats typically decreases with environmental and object familiarity, and the relative levels of exploratory behavior have been used by others as a measure of object/environment recognition (Save et al. 1992; Lee et al. 2005a; Mumby et al. 2007). In our study rats spent more time moving around (exploration) in an arena that contained no objects on the first exposure than on the fifth exposure. Thus, rats displayed behavioral habituation upon repeated exposure to an arena. Rats spent even more time exploring an arena on the fifth exposure when it contained five novel objects than did rats exposed to an arena for the first time (with no objects) or for the fifth time when it contained one novel object, suggesting an additional effect of environmental complexity on the amount of ambulatory behavior.

The relative Fos expression levels associated with the different treatment groups displayed a different pattern than the relative levels of exploratory behavior associated with the different treatment groups. In most brain regions examined Fos expression levels were elevated to comparable levels in rats exposed to the arena, regardless of whether it was the first or fifth exposure or whether the arena contained zero, one, or five novel objects on the test day. This was even the case in the primary somatosensory and motor cortex despite the fact that behaviorally the rats displayed significant differences in ambulatory behavior between some of these conditions, as described above. Presumably, the amount of ambulatory behavior was positively associated with the amount of whisker activity and somatosensory input to the barrel fields (the primary sensory cortical region for rat whisker sensation). A positive correlation has been found for the amount of $c$-fos mRNA in the barrel fields and the intensity of whisker stimulation via magnetic field manipulation of wire filaments attached to individual whiskers (Melzer and Steiner 1997). Similarly, a positive correlation has been observed between the electrophysiological activity of the primary motor cortex and muscle activity (Brecht et al. 2004). Apparently, however, the different 


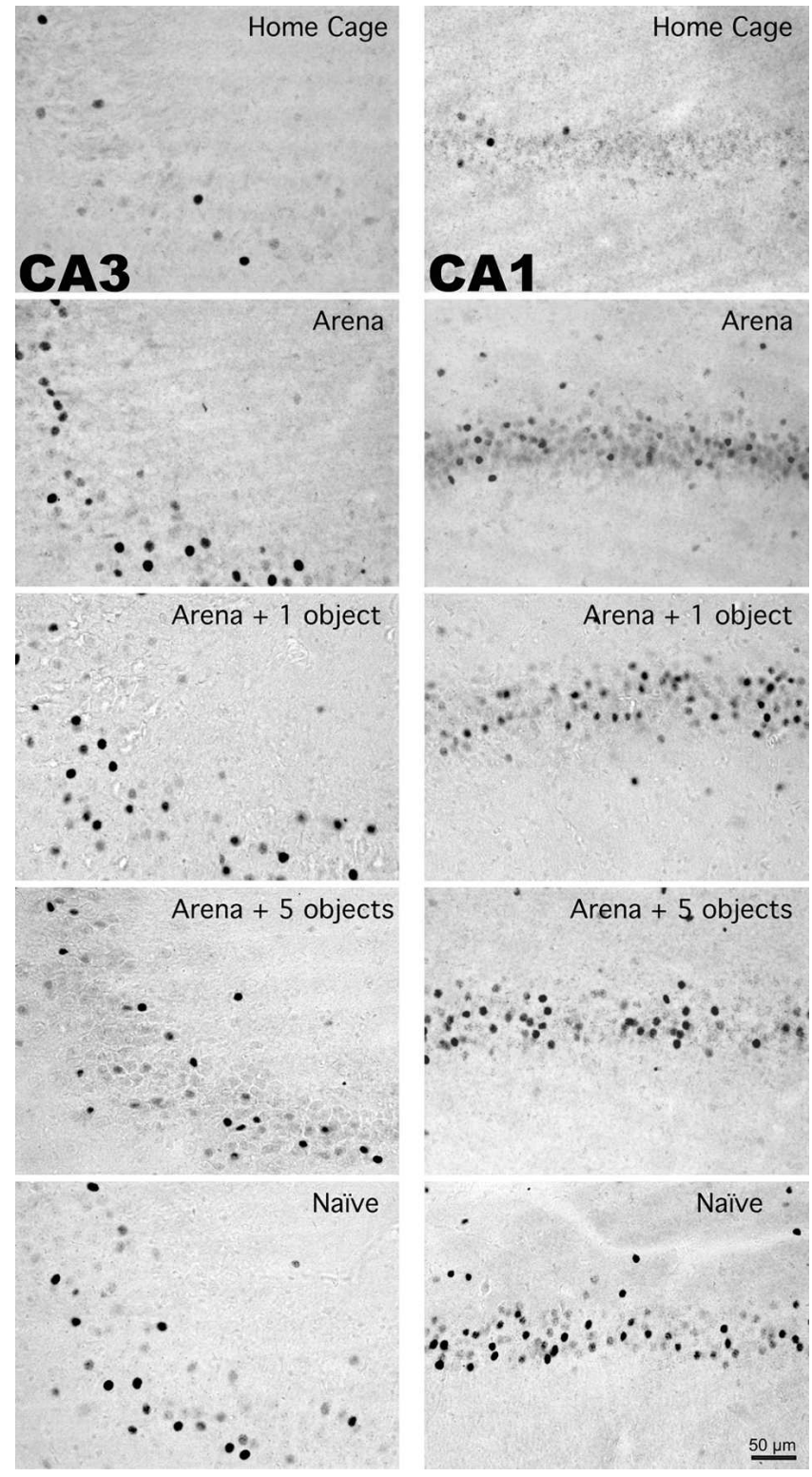

Figure 5. Representative photomicrographs of Fos immunoreactivity within the CA3 and CA1 hippocampal subregions of individual rats exposed to varying amounts of novelty. Note the increased number of Fos immunoreactive cells in the CA1, but not CA3, hippocampal subregion of rats placed in an arena for the first time (Naive) or fifth time with five novel objects (Arena + five objects) compared to rats placed in an arena for the fifth time with zero or one novel object (Arena, Arena + one object, respectively).

amounts of motor and somatosensory activity that result from exploration of an arena (and objects within that arena) are not associated with different numbers of Fos immunoreactive cells within the primary motor and somatosensory cortex.

It is noteworthy that in our second experiment there were three brain regions, the hippocampal CA1 subregion, layer five of the lateral ERC, and the perirhinal cortex in which novelty was associated with a significant increase in the number of Fos immunoreactive cells. Thus, in those three brain regions there were a greater number of Fos immunoreactive cells in rats exposed to the arena for the first time compared to the fifth time. This difference was especially pronounced in the CA1 subregion. Moreover, in the CA1 subregion there appeared to be a novelty "dose-
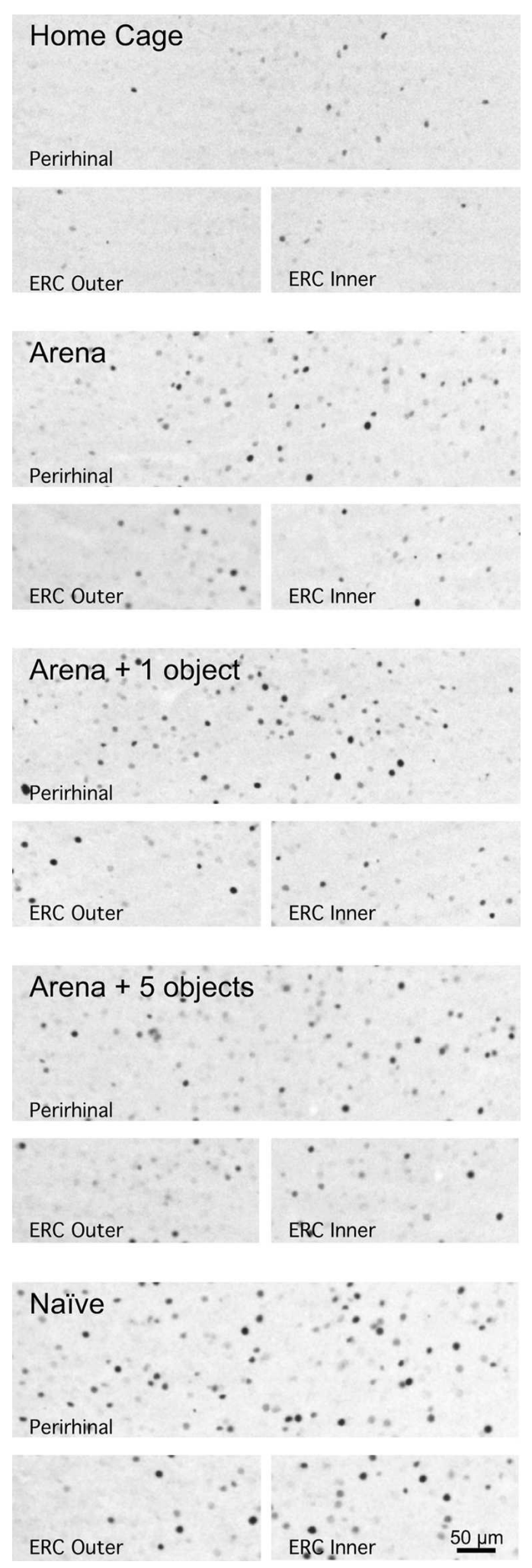

Figure 6. Representative photomicrographs of Fos immunoreactivity within the perirhinal cortex and outer (layer two) and inner (layer five) layers of entorhinal cortex (ERC) of individual rats exposed to varying amounts of novelty. Note the increased number of Fos immunoreactive cells in the inner layer of ERC and perirhinal cortex, but not in the outer layer of ERC of rats placed in an arena for the first time (Naïve) compared to rats placed in an arena for the fifth time (Arena). 


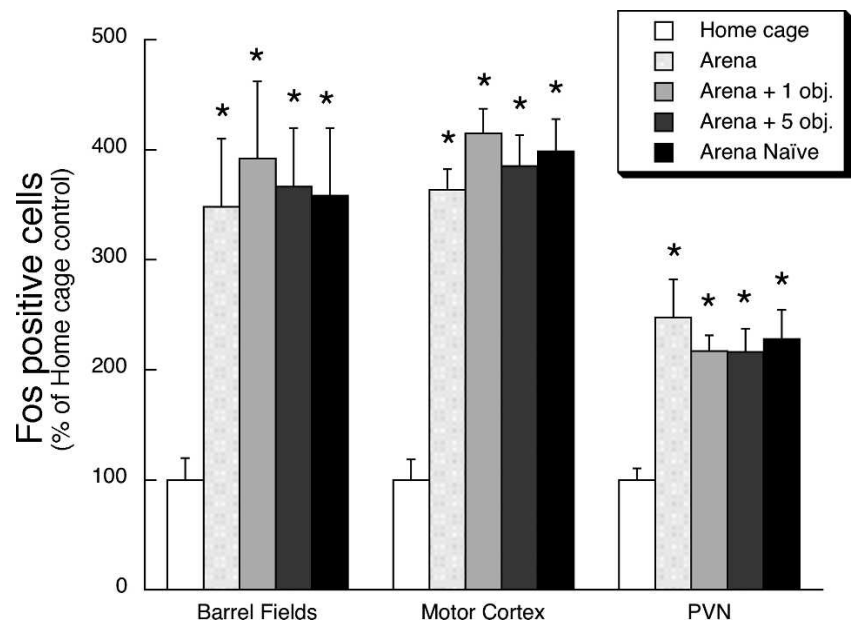

Figure 7. Effect of first time placement in arena or fifth time placement in arena (with zero, one, or five novel objects) on relative Fos expression in select brain regions outside the hippocampal formation. Rats were placed for the first time in a circular arena that contained zero objects on its floor ("Naïve" group), or fifth time in the arena with zero, one, or five novel objects on the floor. After arena exposure (30 min) rats were returned to their home cage for an additional $120 \mathrm{~min}$. Control rats remained in their home cage. Relative Fos expression was examined within a subregion of primary somatosensory cortex (Barrel Fields), primary motor cortex, and the paraventricular nucleus of the hypothalamus (PVN). ${ }^{*} P<0.01$, compared to home-cage control treatment group ( $\mathrm{N}-\mathrm{K}$ test).

response" relationship, with a graded increase in the number of Fos immunoreactive cells in rats re-exposed to an arena that contained zero, one, or five novel objects, respectively, on the test day. This observation is supported by the fact that within the CA1 subregion there was a strong correlation between Fos expression and the manipulated amount of environment novelty present during the test condition. Somewhat surprisingly, there was no statistically significant correlation between Fos expression patterns and ambulatory behavior on the test day in any of the brain regions examined. Perhaps hippocampal encoding of environmental novelty is not closely coupled with the absolute ambulatory activity of the rat as it physically engages with the environment.

The selective association of Fos induction with novelty in CA1 and layer five of the entorhinal cortex has some interesting implications given the known neural connections of the hippocampal formation as described in the introduction. CA1 neurons may serve as comparators for cortically derived information that has and has not been processed by the hippocampal trisynaptic circuit. CA1 neurons also provide the primary cortical output from the hippocampus. A majority of this output is relayed through the subiculum and layer five of the entorhinal cortex (Witter and Amaral 2004). Consequently, activity of CA1 neurons and their target cells (entorhinal cortex layer five) are likely to reflect emergent neural processing of the hippocampus. Based on a variety of experimental evidence, researchers believe that an important function of the hippocampus is novelty detection, especially as it relates to novel associations between experiential elements (Hasselmo and Schnell 1994; Knight 1996; Zhu et al. 1997; Moser and Paulsen 2001; Vinogradova 2001; Meeter et al. 2004; Kumaran and Maguire 2007). Results from our study suggest that a manifestation of novelty detection is increased Fos expression within CA1 and entorhinal cortex layer five. The Fos expression patterns that we observed may or may not indicate that a greater number of neurons in these subregions are electrophysiologically active as a result of novelty (Labiner et al. 1993). Nevertheless, several studies observed a higher firing rate of CA1 pyramidal cells associated with novel environmental or task features (Otto and Eichenbaum 1992; Fyhn et al. 2002; Nitz and McNaughton 2004). Our study demonstrates that as a result of novelty, more cells in the CA1 and entorhinal cortex layer five are engaged in a manner sufficient to produce increased Fos expression. Based on this evidence we conclude that some aspect of novelty detection must originate within the hippocampus since novelty-associated differences in Fos expression were observed in hippocampal output, but not input, elements. The functional significance of greater Fos induction remains to be determined, but given its role as a transcription factor, it is likely to contribute to transcriptionally dependent neuroplasticity (Sheng and Greenberg 1990). This neuroplasticity-supporting molecular event is not necessarily accompanied by parallel changes in expression patterns of other immediate early genes. Guzowski et al. (2006) found that the amount of experience-induced Arc gene expression in hippocampal CA1 neurons does not change across daily open-field exposure sessions repeated over $9 \mathrm{~d}$, but instead decrements when sessions are spaced closely together (intersession intervals 25-55 $\mathrm{min}$ ).

Several other studies have also observed an increase of Fos expression within the CA1 hippocampal subregion with other conditions of novelty (Wan et al. 1999; Vann et al. 2000). However, other factors in addition to novelty are likely to produce CA1 selective increases in Fos expression. One study found a CA1 selective increase in Fos expression with a more explicit learning situation-re-exposure of mice to a conditioned fear context (Strekalova et al. 2003). It should also be noted that novelty per se may not be sufficient to induce Fos in the CA1 subdivision. Pace et al. (2005) found substantially less Fos immunoreactivity and c-fos mRNA in the hippocampus of rats exposed for the first time to restraint than to an arena. Other studies noted that novel visual objects or novel gustatory stimuli were not very effective at inducing hippocampal Fos expression (Zhu et al. 1997; MontagSallaz et al. 1999; Wan et al. 1999), whereas, a novel configuration of visual objects was effective (Wan et al. 1999). Therefore, increased CA1 hippocampal Fos expression may be selectively related to novel situations that specifically engage the hippocampus, such as spatial configural novelty. Interestingly, Fyhn et al. (2002) recorded CA1 electrophysiological activity of rats in a water maze and found that activity increased with novel platform location, but only when the rat had been trained to locate a

Table 1. Pearson correlation coefficients for linear relationship between Fos-positive cells within each brain region of interest and test day ambulatory activity or novelty rank of the test day condition

\begin{tabular}{lcc}
\hline Brain region & Total ambulation & Test condition novelty rank \\
\hline ERC outer & -0.17 & 0.11 \\
DG-Supra & -0.09 & 0.29 \\
DG-Infra & -0.27 & -0.06 \\
CA4 & 0.30 & 0.20 \\
CA3 & 0.14 & 0.29 \\
CA2 & 0.18 & -0.22 \\
CA1 & 0.28 & $0.61^{\mathrm{a}}$ \\
ERC inner & 0.14 & $0.36^{\mathrm{b}}$ \\
Perirhinal & 0.15 & $0.39^{\mathrm{b}}$ \\
Barrel fields & 0.32 & 0.01 \\
Motor cortex & 0.06 & 0.12 \\
PVN & 0.08 & 0.07
\end{tabular}

Novelty ranks were as follows: fifth exposure to the arena $=1$, fifth exposure to the arena with one novel object $=2$, fifth exposure to the arena with five novel objects $=3$, and first exposure to the arena $=4$.

a $P<0.002$ (critical $P$ applying Bonferroni correction for multiple comparisons).

${ }^{\mathrm{b}} \mathrm{P}<0.05$. 
different specific platform location. Thus, expectations may contribute to the extent to which novelty alters CA1 hippocampal neuron activity.

Some other studies and models support a specific role of the CA3 subregion and dentate gyrus in novelty detection (Hess et al. 1995b; Meeter et al. 2004). Few studies have examined the effects of selective lesions of hippocampal subregions on noveltydependent behavior. In general, studies in which a substantial portion of the dorsal hippocampus (or its primary efferents) is lesioned support a functional role of the hippocampus in detection of novel spatial relationships, but not novel objects (Save et al. 1992; Mumby and Pinel 1994; Honey et al. 1998; Bussey et al. 2000; Mumby et al. 2002). Of the few studies that have examined the effects of lesions of hippocampal subregions, Lee et al. (2005a) found that neurotoxic lesion of dorsal dentate gyrus or CA3 produced a greater impairment of spatial novelty-dependent exploratory behavior than did lesion of CA1. However, the same group has found that lesion of the CA1 subregion produces greater impairment than lesion of CA3 or dentate gyrus on tasks that depend on recall of the temporal order in which spatial locations were visited (Gilbert et al. 2001; Lee et al. 2005b). Our results do not preclude the involvement of other hippocampal subregions in environmental novelty detection, but changes in CA3 and dentate gyrus neural activity as a result of the novel features of our experimental conditions were not sufficient to alter the overall number of Fos immunoreactive cells within those subregions.

The perirhinal cortex may also play a role in novelty detection, but one that differs from the hippocampal formation. Neuronal activity in the perirhinal cortex appears to register novel exposure to objects that is independent of their association with other objects or contexts (Kumaran and Maguire 2007). Moreover, there is some evidence that this object novelty detection occurs independent of hippocampal formation function (Wan et al. 1999; Brown and Aggleton 2001). Interestingly, in this study we found that first time exposure to a circular arena induced greater Fos expression in the perirhinal cortex than did the fifth exposure. Thus, this novel experience in the absence of discrete object stimuli was sufficient to induce greater Fos expression in the perirhinal cortex, and this response was attenuated by the fifth exposure. Whether this novelty related Fos expression was independent of the parallel novelty related Fos expression observed in the output components of the hippocampal formation remains to be determined. The subregion of perirhinal cortex (rostral-ventral region) that we examined has a fairly extensive reciprocal connection with the lateral entorhinal cortex, but very little direct connection with any other component of the hippocampal formation (Furtak et al. 2007).

The greater Fos expression in CA1 and layer five of the entorhinal cortex associated with novelty does not appear to be secondary to some general arousal level that may have resulted from the heightened stress/anxiety of novelty. The arena exposure did not appear to be very stressful for any of the treatment conditions. Novel arena or object exposure produced an increase rather than decrease in exploratory behavior, with decreased behavior expected if there was high anxiety and freezing behavior. In addition, no differences in Fos expression in the PVN were observed between the different treatment groups, suggesting comparable levels of hypothalamic-pituitary-adrenal axis activation (Pace et al. 2005).

In contrast to all other brain regions examined, we observed a decrease in the number of Fos immunoreactive cells in the infrapyramidal blade of the dentate gyrus upon exposure to the arena. This is a robust phenomenon that we and others have previously observed after exposure to a variety of novel experiences (Chowdhury et al. 2000; Fevurly and Spencer 2004; Pace et al. 2005). Historically, no distinctions in the functional, structural, and neural innervation of the two blades of the dentate gyrus have been emphasized. However, more recent studies have identified a number of neurochemical, neuroanatomical, and functional differences between the two blades (Hartmann et al. 1992; Tamamaki 1997; Scharfman et al. 2002; Choi et al. 2003; Kim et al. 2004; Witter and Amaral 2004).

In summary, first time exposure to a novel environment or novel objects within a familiar environment produced a selective augmentation of Fos expression in dorsal CA1 hippocampal neurons, one of their projection sites, layer five of the lateral entorhinal cortex, and perirhinal cortex. These data support the hypothesis that general hippocampal activity is altered by environmental novelty and the prospect that novelty detection is an emergent process within the hippocampus that is manifest by heightened Fos expression in the cortical output, but not input circuit components. Concurrent novelty-dependent increases in Fos expression in perirhinal cortex may represent altered neural activity relayed from the entorhinal cortex, or it may reflect an independent novelty detection process that also emerges with the particular test conditions used in this study.

\section{Materials and Methods}

\section{Subjects}

Adult male Sprague-Dawley rats were purchased from Harlan (Facility 218, Prattville, AL) and housed in animal facilities at the University of Colorado. Rats were housed two per cage in polycarbonate tubs $(47 \times 23 \times 20 \mathrm{~cm})$ with wood chip bedding in a temperature $\left(22^{\circ} \mathrm{C} \pm 2^{\circ} \mathrm{C}\right)$ and humidity controlled room. Lights were maintained on a 12:12 h light/ dark cycle (lights on 07.00 h). Food and water were continuously available except during the time of experimentation. Rats were given 2 wk to acclimate to the animal facility prior to exposure to experimental procedures. All procedures were approved by the University of Colorado at Boulder Institutional Animal Care and Use Committee.

\section{Novel environment and objects}

Arena exposure consisted of placing a rat on the floor of the test room in the center of a large circular sheet metal enclosure (2-m diameter, 1-m-high wall). The floor inside the arena was cleaned with a damp towel and dried before each rat was placed in the arena. For some rats on the experimental test day the arena included placement of one or five different objects on the floor of the arena. Objects consisted of two small plastic toys, a set of keys, a paperweight, and an empty pipet-tip box. Each item was heavy enough that the rats could not easily push them around on the arena floor. Objects were distributed evenly around the arena, approximately midway between the center and perimeter wall. For each session rats were placed directly in the center of the arena. The arena containing room was adjacent to, but separate from the home-cage room.

\section{Experiment 1 procedure: Variation in environmental complexity}

Rats were randomly divided into four treatment groups $(n=4)$. Three groups were placed into an arena for the first time, and the groups differed in whether the arena contained zero, one, or five objects on its floor. Thirty minutes after arena exposure rats were returned to their home cage, and then 120 min later were deeply anesthetized for transcardial perfusion. The fourth group (Control group) remained undisturbed in their home cage until they were deeply anesthetized for transcardial perfusion. Arena exposure occurred between 0900 and $1200 \mathrm{~h}$.

\section{Experiment 2 procedure: Variation in environmental novelty}

Rats were randomly divided into five treatment groups $(n=8)$ that varied in terms of the number of days of arena exposure 
(zero, one, or five) and in the number of novel objects present in the arena on the test day (zero, one, or five). The experiment was conducted over five consecutive days. Three groups of rats were placed in an arena that had no objects on the floor for $30 \mathrm{~min}$ a day for four consecutive days. On the fifth day (test day), rats were returned to an arena (30 $\mathrm{min})$ that contained zero (Arena group), one (Arena + one object group), or five (Arena + five objects group) novel objects. Two additional groups of rats (Control group and Naïve group) remained in their home cages until the test day. On the test day Naïve rats were given, for the first time, 30 min of exposure to an arena that did not contain objects. Control rats remained undisturbed in their home cage until they were deeply anesthetized for transcardial perfusion. Rats in the other four treatment groups were returned to their home cage after arena exposure, and then 120 min later were deeply anesthetized for transcardial perfusion. Arena exposure occurred between 0900 and $1300 \mathrm{~h}$ each day, and the time of day for arena exposure was counterbalanced across treatment groups. This experiment was conducted with two separate cohorts of rats and the same procedure was followed for each cohort (with four rats per treatment group in each cohort).

\section{Ambulatory behavior}

Videotapes of the test day session from the second experiment were scored for general ambulatory activity. The person scoring the videotapes used a stopwatch to measure the total amount of time that each rat was engaged in ambulatory movement during the first and second 10-min intervals after placement in the arena. Ambulatory movement was defined as movement of all four limbs from one place to another, rather than simple head movements, rearing, sniffing, or stretching.

\section{Tissue preparation and immunohistochemistry}

At the appropriate experimental time point, rats were deeply anaesthetized (75 mg/kg ketamine/15 mg/kg xylazine i.p.) and transcardially perfused with $400 \mathrm{~mL}$ of heparinized $(1 \mathrm{unit} / \mathrm{mL})$ $0.01 \mathrm{M}$ phosphate buffer followed by $400 \mathrm{~mL}$ of $4 \%$ paraformaldehyde in phosphate buffer. After extraction, brains were postfixed in $4 \%$ paraformaldehyde phosphate buffer at $4^{\circ} \mathrm{C}$ for $\sim 48 \mathrm{~h}$. Brains were sectioned (50 $\mu \mathrm{m}$ thick coronal sections) with a vibratome (Vibratome Co.). Fos protein was visualized using a standard avidin-biotin-horseradish peroxidase immunohistochemical procedure. Tissue sections were incubated overnight in a rabbit polyclonal anti-c-Fos antibody (sc-52, Santa Cruz Biotechnology; 1:7500 dilution) that, according to the supplier, does not cross-react with other Fos-related gene products such as Fos B, Fra-1, or Fra-2. After washing in assay buffer $(0.01 \mathrm{M}$ sodium phosphate), sections were incubated in biotinylated goat antirabbit antibody (Vector Laboratories Inc.; 1:750 dilution) followed by incubation in an avidin-biotin-horseradish peroxidase solution. Diaminobenzidine (DAB, $0.2 \mathrm{mg} / \mathrm{mL}$ in the presence of $7 \mathrm{mg} / \mathrm{mL}$ nickel ammonium sulfate) was used as chromogen. To allow for a direct comparison of the number of immunopositive cells between different brains, all brain sections from a given experimental cohort were processed simultaneously in the same pool of each reagent using staining net dishes (Brain Research Laboratories).

\section{Fos-positive cell counts}

The number of Fos-positive cells was counted using a computerized image analysis system (Olympus Microsuite Analysis 3.2; Soft Imaging System Corp.). A threshold gray level for positive cells that was $\sim 50 \%$ of the maximum gray level signal over background was chosen. For a given brain region, four to six separate counts taken from separate sections/hemispheres were conducted for each brain and were then averaged to yield a final value for that brain. Brain sections to be analyzed were coded so that cell count analysis was performed by an individual who did not know the treatment group assignment for each section.

Sections (six per brain) for analysis of Fos immunoreactivity in the dorsal hippocampus, dentate gyrus, lateral entorhinal cortex, and perirhinal cortex were centered approximately around
$3.6 \mathrm{~mm}$ posterior to bregma. Within the hippocampus, cell counts were performed over the pyramidal cell body layer extending from subregions CA1-CA4. The rat brain atlas of Paxinos and Watson (1998) was used as a visual guide for determining subregion boundaries (Fig. 1). The CA4 region was designated as the hippocampal principal cell layer that was located between the two blades of the dentate gyrus. Within the dentate gyrus, separate cell counts were performed over the granule cell body layer in the suprapyramidal (inner) blade and the infrapyramidal (outer) blade. To control for the slight differences in hippocampal brain region cross-sectional area, Fos-positive cell counts were expressed as the number of cells per subregion square micrometer. Within the lateral entorhinal cortex a uniform rectangular region of interest was centered over a relatively dense cell body layer close to the lateral surface (approximately cortical layer two) and a second uniform rectangular region of interest was centered over a relatively dense cell body layer close to the amygdalar capsule (approximately cortical layer five). Within the perirhinal cortex there was a relatively uniform distribution of Fospositive cells across all of the deeper cortical layers, with very few Fos-positive cells evident in the superficial portion of perirhinal cortex. A rectangular region of interest was centered over the Fos-positive cell containing deeper layers of perirhinal cortex. For the PVN, immunopositive cells were counted within the entire nucleus including both parvocellular and magnocellular neurons (centered $\sim 1.8 \mathrm{~mm}$ posterior to bregma). The majority of Fos immunopositive cells, however, were localized to the medial parvocellular portion of the nucleus (data not shown). On the same coronal sections that contained the PVN, Fos immunopositive cell counts were determined for the primary motor cortex and the primary somatosensory cortex (barrel field), using a uniform rectangular region of interest that spanned all cortical layers.

\section{Statistical analysis}

In order to provide for a direct comparison of relative Fos immunoreactivity between experiments 1 and 2 and between brain regions within an experiment, data were transformed as a percent of the mean value for the home-cage Control group. Fos data were initially analyzed for each brain region with separate one-way analysis of variance (ANOVA) tests. Unless indicated otherwise, the Student-Newman-Keuls multiple comparison test $(\mathrm{N}-\mathrm{K})$ was used to examine pairwise differences between treatment groups for analyses in which there was an overall treatment effect $(\alpha$-level $<0.05)$.

For the second experiment, ambulatory activity of rats placed in an arena ( \pm novel objects) on the test day was scored. Because the overall levels of ambulatory activity varied between the two cohorts, ambulatory activity was transformed as a percent of the mean value for the first 10-min interval of the Arena group of each cohort. Analysis (ANOVA) of pooled behavioral data in Experiment 2 that included a cohort factor found no significant cohort by treatment interaction. Ambulatory behavior was analyzed with a mixed design two-way ANOVA (treatment group as a between-group variable, and test day time interval as a within-group variable). The Student-Newman-Keuls multiple comparison test was used to examine pairwise differences between treatment groups $(\alpha$-level $<0.05)$.

A correlational analysis (Pearson correlation) for the second experiment was also conducted to determine if there was a linear relationship between the number of Fos-positive cells within a particular brain region and either ambulatory behavior of rats or the extent of novelty present during the test day condition. The extent of novelty present was assigned a rank value of $1-4$, with exposure to the arena for the fifth time (containing zero objects) considered least novel $(\mathrm{rank}=1)$, exposure to the arena for the first time (containing zero objects) considered most novel $($ rank $=4)$, and exposure to the arena for the fifth time with one or five novel objects considered to have intermediate levels of novelty (rank $=2$ and 3 , respectively).

A computer graphing and statistical package (KaleidaGraph, v 4.02, Synergy Software) was used to perform the analyses. Data presented in the figures represent group means \pm SEM. 


\section{Acknowledgments}

We are very grateful to Jerry Rudy for thoughtful discussion and critique of these experiments and preliminary versions of the manuscript. This work was supported by National Institutes of Health grants (MH 62456 and MH 65977) and by the University of Colorado Undergraduate Research Opportunity Program.

\section{References}

Andersen, P., Bliss, T.V.P., and Skrede, K.K. 1971. Lamellar organization of hippocampal excitatory pathways. Exp. Brain Res. 13: 222-238.

Brecht, M., Schneider, M., Sakmann, B., and Margrie, T.W. 2004. Whisker movements evoked by stimulation of single pyramidal cells in rat motor cortex. Nature 427: 704-710.

Brown, M.W. and Aggleton, J.P. 2001. Recognition memory: What are the roles of the perirhinal cortex and hippocampus? Nat. Rev. Neurosci. 2: 51-61.

Bussey, T.J., Duck, J., Muir, J.L., and Aggleton, J.P. 2000. Distinct patterns of behavioural impairments resulting from fornix transection or neurotoxic lesions of the perirhinal and postrhinal cortices in the rat. Behav. Brain Res. 111: 187-202.

Choi, Y.S., Lee, M.Y., Sung, K.W., Jeong, S.W., Choi, J.S., Park, H.J., Kim, O.N., Lee, S.B., and Kim, S.Y. 2003. Regional differences in enhanced neurogenesis in the dentate gyrus of adult rats after transient forebrain ischemia. Mol. Cells 16: 232-238.

Chowdhury, G.M., Fujioka, T., and Nakamura, S. 2000. Induction and adaptation of Fos expression in the rat brain by two types of acute restraint stress. Brain Res. Bull. 52: 171-182.

Fevurly, R.D. and Spencer, R.L. 2004. Fos expression is selectively and differentially regulated by endogenous glucocorticoids in the paraventricular nucleus of the hypothalamus and the dentate gyrus. J. Neuroendocrinol. 16: 970-979.

Furtak, S.C., Wei, S.M., Agster, K.L., and Burwell, R.D. 2007. Functional neuroanatomy of the parahippocampal region in the rat: The perirhinal and postrhinal cortices. Hippocampus 17: 709-722.

Fyhn, M., Molden, S., Hollup, S., Moser, M.B., and Moser, E. 2002. Hippocampal neurons responding to first-time dislocation of a target object. Neuron 35: 555-566.

Gilbert, P.E., Kesner, R.P., and Lee, I. 2001. Dissociating hippocampal subregions: Double dissociation between dentate gyrus and CA1. Hippocampus 11: 626-636.

Guzowski, J.F., Miyashita, T., Chawla, M.K., Sanderson, J., Maes, L.I., Houston, F.P., Lipa, P., McNaughton, B.L., Worley, P.F., and Barnes, C.A. 2006. Recent behavioral history modifies coupling between cell activity and Arc gene transcription in hippocampal CA1 neurons. Proc. Natl. Acad. Sci. 103: 1077-1082.

Hartmann, D., Sievers, J., Pehlemann, F.W., and Berry, M. 1992. Destruction of meningeal cells over the medial cerebral hemisphere of newborn hamsters prevents the formation of the infrapyramidal blade of the dentate gyrus. J. Comp. Neurol. 320: 33-61.

Hasselmo, M.E. and Schnell, E. 1994. Laminar selectivity of the cholinergic suppression of synaptic transmission in rat hippocampal region CA1: Computational modeling and brain slice physiology. J. Neurosci. 14: 3898-3914.

Herdegen, T. and Leah, J.D. 1998. Inducible and constitutive transcription factors in the mammalian nervous system: Control of gene expression by Jun, Fos and Krox, and CREB/ATF proteins. Brain Res. Brain Res. Rev. 28: 370-490.

Hess, U.S., Lynch, G., and Gall, C.M. 1995a. Regional patterns of c-fos mRNA expression in rat hippocampus following exploration of a novel environment versus performance of a well-learned discrimination. J. Neurosci. 15: 7796-7809.

Hess, U.S., Lynch, G., and Gall, C.M. 1995b. Changes in c-fos mRNA expression in rat brain during odor discrimination learning: Differential involvement of hippocampal subfields CA1 and CA3. J. Neurosci. 15: 4786-4795.

Honey, R.C., Watt, A., and Good, M. 1998. Hippocampal lesions disrupt an associative mismatch process. J. Neurosci. 18: 2226-2230.

Johnson, J.D., Muftuler, L.T., and Rugg, M.D. 2008. Multiple repetitions reveal functionally and anatomically distinct patterns of hippocampal activity during continuous recognition memory. Hippocampus 18: 975-980.

Kim, S.Y., Min, D.S., Choi, J.S., Choi, Y.S., Park, H.J., Sung, K.W., Kim, J., and Lee, M.Y. 2004. Differential expression of phospholipase D isozymes in the hippocampus following kainic acid-induced seizures. J. Neuropathol. Exp. Neurol. 63: 812-820.

Knight, R. 1996. Contribution of human hippocampal region to novelty detection. Nature 383: 256-259.

Kumaran, D. and Maguire, E.A. 2007. Which computational mechanisms operate in the hippocampus during novelty detection? Hippocampus 17: 735-748.
Labiner, D.M., Butler, L.S., Cao, Z., Hosford, D.A., Shin, C., and McNamara, J.O. 1993. Induction of c-fos mRNA by kindled seizures: Complex relationship with neuronal burst firing. J. Neurosci. 13: 744-751.

Lee, I., Hunsaker, M.R., and Kesner, R.P. 2005a. The role of hippocampal subregions in detecting spatial novelty. Behav. Neurosci. 119: 145-153.

Lee, I., Jerman, T.S., and Kesner, R.P. 2005b. Disruption of delayed memory for a sequence of spatial locations following CA1- or CA3-lesions of the dorsal hippocampus. Neurobiol. Learn. Mem. 84: 138-147.

Margulies, D.M. 1985. Selective attention and the brain: A hypothesis concerning the hippocampal-ventral striatal axis, the mediation of selective attention, and the pathogenesis of attentional disorders. Med. Hypotheses 18: 221-264.

Meeter, M., Murre, J.M., and Talamini, L.M. 2004. Mode shifting between storage and recall based on novelty detection in oscillating hippocampal circuits. Hippocampus 14: 722-741.

Melzer, P. and Steiner, H. 1997. Stimulus-dependent expression of immediate-early genes in rat somatosensory cortex. J. Comp. Neurol. 380: $145-153$.

Mizumori, S.J., Ragozzino, K.E., Cooper, B.G., and Leutgeb, S. 1999. Hippocampal representational organization and spatial context. Hippocampus 9: 444-451.

Montag-Sallaz, M., Welzl, H., Kuhl, D., Montag, D., and Schachner, M. 1999. Novelty-induced increased expression of immediate-early genes c-fos and arg 3.1 in the mouse brain. J. Neurobiol. 38: 234-246.

Moser, E.I. and Paulsen, O. 2001. New excitement in cognitive space: Between place cells and spatial memory. Curr. Opin. Neurobiol. 11: $745-751$.

Mumby, D.G. and Pinel, J.P. 1994. Rhinal cortex lesions and object recognition in rats. Behav. Neurosci. 108: 11-18.

Mumby, D.G., Gaskin, S., Glenn, M.J., Schramek, T.E., and Lehmann, H. 2002. Hippocampal damage and exploratory preferences in rats: Memory for objects, places, and contexts. Learn. Mem. 9: 49-57.

Mumby, D.G., Piterkin, P., Lecluse, V., and Lehmann, H. 2007. Perirhinal cortex damage and anterograde object-recognition in rats after long retention intervals. Behav. Brain Res. 185: 82-87.

Nitz, D. and McNaughton, B. 2004. Differential modulation of CA1 and dentate gyrus interneurons during exploration of novel environments. J. Neurophysiol. 91: 863-872.

Norman, K.A. and O'Reilly, R.C. 2003. Modeling hippocampal and neocortical contributions to recognition memory: A complementary-learning-systems approach. Psychol. Rev. 110: 611-646.

Otto, T. and Eichenbaum, H. 1992. Neuronal activity in the hippocampus during delayed non-match to sample performance in rats: Evidence for hippocampal processing in recognition memory. Hippocampus 2: 323-334.

Pace, T.W., Gaylord, R., Topczewski, F., Girotti, M., Rubin, B., and Spencer, R.L. 2005. Immediate-early gene induction in hippocampus and cortex as a result of novel experience is not directly related to the stressfulness of that experience. Eur. J. Neurosci. 22: 1679-1690.

Paxinos, G. and Watson, C. 1998. The rat brain in stereotaxic coordinates, 4 th ed. Academic, San Diego, CA.

Remondes, M. and Schuman, E.M. 2004. Role for a cortical input to hippocampal area CA1 in the consolidation of a long-term memory. Nature 431: 699-703.

Rudy, J.W. and Sutherland, R.J. 1995. Configural association theory and the hippocampal formation: An appraisal and reconfiguration. Hippocampus 5: 375-389.

Save, E., Poucet, B., Foreman, N., and Buhot, M.C. 1992. Object exploration and reactions to spatial and nonspatial changes in hooded rats following damage to parietal cortex or hippocampal formation. Behav. Neurosci. 106: 447-456.

Scharfman, H.E., Sollas, A.L., Smith, K.L., Jackson, M.B., and Goodman, J.H. 2002. Structural and functional asymmetry in the normal and epileptic rat dentate gyrus. J. Comp. Neurol. 454: 424-439.

Sheng, M. and Greenberg, M.E. 1990. The regulation and function of c-fos and other immediate early genes in the nervous system. Neuron 4: 477-485.

Stern, C.E., Corkin, S., González, R.G., Guimaraes, A.R., Baker, J.R., Jennings, P.J., Carr, C.A., Sugiura, R.M., Vedantham, V., and Rosen, B.R. 1996. The hippocampal formation participates in novel picture encoding: Evidence from functional magnetic resonance imaging. Proc. Natl. Acad. Sci. 93: 8660-8665.

Steward, O. and Scoville, S.A. 1976. Cells of origin of entorhinal cortical afferents to the hippocampus and fascia dentata of the rat. J. Comp. Neurol. 169: 347-370.

Strekalova, T., Zörner, B., Zacher, C., Sadovska, G., Herdegen, T., and Gass, P. 2003. Memory retrieval after contextual fear conditioning induces c-Fos and JunB expression in CA1 hippocampus. Genes Brain 
Behav. 2: 3-10.

Tamamaki, N. 1997. Organization of the entorhinal projection to the rat dentate gyrus revealed by Dil anterograde labeling. Exp. Brain Res. 116: $250-258$

Vann, S.D., Brown, M.W., Erichsen, J.T., and Aggleton, J.P. 2000. Fos imaging reveals differential patterns of hippocampal and

parahippocampal subfield activation in rats in response to different spatial memory tests. J. Neurosci. 20: 2711-2718.

Vinogradova, O.S. 2001. Hippocampus as comparator: Role of the two input and two output systems of the hippocampus in selection and registration of information. Hippocampus 11: 578-598.

Wan, H., Aggleton, J.P., and Brown, M.W. 1999. Different contributions of the hippocampus and perirhinal cortex to recognition memory. J.
Neurosci. 19: 1142-1148.

Witter, M. and Amaral, D. 2004. Hippocampal formation. In The rat nervous system, 3rd ed. (ed. G. Paxinos), pp. 635-704. Elsevier, Amsterdam.

Zhu, X.O., McCabe, B.J., Aggleton, J.P., and Brown, M.W. 1997. Differential activation of the rat hippocampus and perirhinal cortex by novel visual stimuli and a novel environment. Neurosci. Lett. 229. $141-143$.

Received August 21, 2008; accepted in revised form October 7, 2008. 


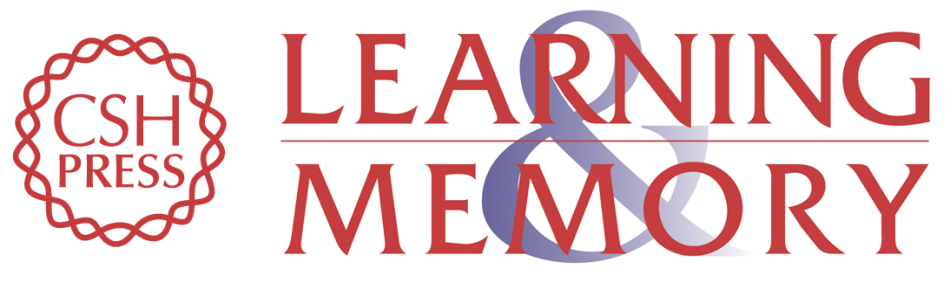

Environmental novelty is associated with a selective increase in Fos expression in the output elements of the hippocampal formation and the perirhinal cortex

Michael VanElzakker, Rebecca D. Fevurly, Tressa Breindel, et al.

Learn. Mem. 2008, 15:

Access the most recent version at doi:10.1101//m.1196508

References This article cites 50 articles, 10 of which can be accessed free at: http://learnmem.cshlp.org/content/15/12/899.full.html\#ref-list-1

License

Email Alerting

Receive free email alerts when new articles cite this article - sign up in the box at the Service top right corner of the article or click here. 